Case Report : Open Access

\title{
Drug-facilitated Crime: A Diagnosis to Remember in the Emergency Department
}

\author{
Rocca $F D^{1}$, Pignatiello $F^{1}$, Casacanditella $G^{1}$, Tucci $M^{2}$ and Favretto $D^{2 *}$ \\ ${ }^{1}$ Emergency Department, University Hospital of Padova, Padova, Italy \\ ${ }^{2}$ Forensic Toxicology and Antidoping, University Hospital of Padova, Padova, Italy
}

*Corresponding author: Donata Favretto, Forensic Toxicology and Antidoping, University Hospital of Padova, Via Giustiniani 2, 35128 Padova, Italy, Tel: +39-(0)49-821-8926, Fax: +39-(0)49-821-8927 E-mail: donata.favretto@unipd. it

\begin{abstract}
We report on a case of a young man arrived to the emergency department (ED) of the local hospital in a sleepy, confused state, and exhibiting anterograde amnesia. He reported to have been robbed. Toxicological analysis performed in his blood sample with a general unknown screening procedure including mass spectrometry detection revealed the presence of zolpidem. This case suggests that emergency physicians should consider drugfacilitated crimes in the differential diagnosis of acute confusion state or anterograde amnesia without an evident clinical reason.
\end{abstract}

\section{Keywords}

Acute confusional state, Anterograde amnesia, Drug-facilitated crimes, Zolpidem, Flumazenil

\section{Case Report}

A 33-years-old man arrived to the ED in a sleepy state and with anterograde amnesia. The patient reported to have drunk a coffee offered by a stranger few hours before, afterwards he became totally confused. He was robbed of his belongings but he did not remember what actually happened. At the ED the patient was pale, sweaty and tachycardic; blood pressure, oxygen saturation and body temperature were normal. The neurological examination showed: GSC 14, equal pupils, round and reactive to light, minor unconsciousness state with anterograde amnesia, absence of cranial nerves deficits or motor and sensory deficits. Heart, lung and abdomen examination were normal. There were no traumatic signs. Chest X-ray and cerebral CT were normal. His past medical history was negative and the patient was not taking any medicinal drugs. Clinical blood parameters were normal and the toxicological screening performed at the ED with an immunochemical assay (benzodiazepine, carbamazepine, barbiturates, tricyclic antidepressants) in urine and enzymatic assay (ethanol) in serum were negative. At the same time, only blood was collected and sent for toxicological analysis. Since the persistent sleepy state, endovenous flumazenil 1 $\mathrm{mg} / 10 \mathrm{ml}$ was administered ex-adiuvantibus, producing a sudden improvement of the level of consciousness. However, anterograde amnesia persisted. Toxicological and forensic analysis performed at the forensic toxicology department of the local university by a general unknown screening procedure, based on liquid/liquid extraction and gas chromatography mass spectrometry-(GC-MS) and liquid chromatography-high resolution mass spectrometry (LCHRMS), both in full scan acquisition mode, revealed the presence of zolpidem into blood sample at $180 \mathrm{ng} / \mathrm{mL}$ (Figure 1). The presence of other xenobiotics including zopiclone, gamma-hydroxybutyrate (GHB), ketamine, phencyclidine, antipsychotics, antidepressants, neuroleptics, antihistaminics, benzodiazepines, barbiturates, cannabinoids, cocaine, and opiates, was excluded. Blood ethanol concentration, determined by head space gas chromatography, was negative $(<0.05 \mathrm{~g} / \mathrm{L})$.

\section{Discussion}

The administration of psychoactive substances to victims for facilitating crimes (robberies, sexual assaults, violence...), known as drug-facilitated crime (DFC), is a well known phenomenon [1]. Nevertheless, DFCs are often difficult to identify, both because physicians are not fully aware of the phenomenon and because the toxicological analyses performed in ED or points of care do not detect all the potentially involved substances. Furthermore, when the victim of a DFC is under the amnesic effects of the drug administered, he/ she may not clearly remember the events leading to the assault; the report of a probable assault to a physician or to a police department often occurs one or two days later, when the substance cannot be detected in the urine or blood [2].

The drugs involved are the ones providing amnesic, sedativehypnotic, anxiolytic, narcotic, and muscle relaxant or disinhibitory effects. The most used substances are benzodiazepines, nonbenzodiazepine hypnotics (zolpidem and zopiclone), antihistaminic and neuroleptic drugs, tricyclic antidepressants, GHB, cannabinoids, amphetamines and opiates. Some of those drugs are detectable in blood only for one or two hours post administration, and in urine for less than one day, while others may be detectable in urine for four or more days after the offence [3,4], so that missing well-timed blood and urine sample often results in missing the administered drug(s).

Emergency physicians should consider DFCs in every case of altered mental status or behaviour and anterograde amnesia without an evident clinical reason, and should search for suggestive elements of this in patient's history and examination. In the case history collection, it is important to record as many details as possible

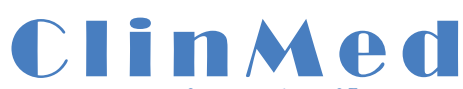
International Library
Citation: Rocca FD, Pignatiello F, Casacanditella G, Tucci M, Favretto D (2016) Drugfacilitated Crime: A Diagnosis to Remember in the Emergency Department. J Toxicol Risk Assess 2:004

Received: December 11, 2015: Accepted: January 21, 2016: Published: January 25, 2016 Copyright: ( 2016 Rocca FD, et al. This is an open-access article distributed under the terms of the Creative Commons Attribution License, which permits unrestricted use, distribution, and reproduction in any medium, provided the original author and source are credited. 

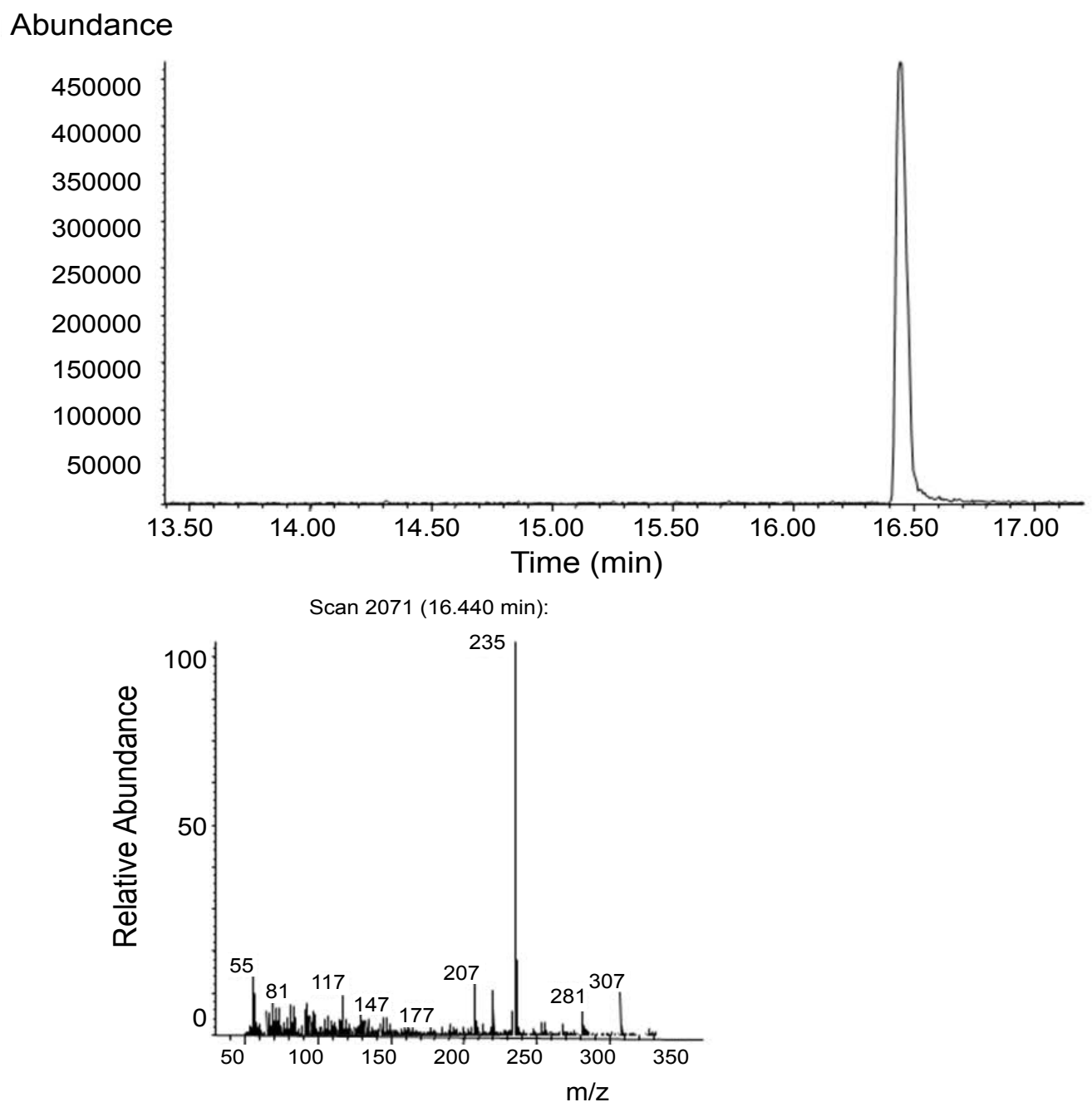

Figure 1: Gas chromatogram and full scan mass spectrum of Zolpidem in the patient blood.

as regards the time and circumstances of the event (a party, an encounter with a stranger, the consumption of drinks or psychoactive substances, etc). It is useful to mark the timing of symptoms onset and the last patient recollection. In our case, the patient reported to have met a "stranger" and this event marked the beginning of his amnesia.

About physical examination, we note that the patient was tachycardic, not a typical zolpidem side effect; we cannot rule out that tachycardia was caused by anxiety and fear, but literature reports tachycardia as rare adverse clinical effect of zolpidem [5,6]. The toxicological screening performed by Forensic Toxicology (included cocaine, cannabinoids, and amphetamines) was negative.

A continuative therapy with psychoactive drugs must also be taken into account for differential diagnosis. The other causes of altered mental status or anterograde amnesia (traumatic, infectious or metabolic diseases) should be ruled out. In our case, blood exams, $\mathrm{X}$-ray chest, cerebral CT and toxicological screening performed in urine (immunoassays for "classical" drugs of abuse) and serum (enzymatic assay for ethanol) at the ED were all negative.

The administration of the classic benzodiazepine antagonist, flumazenil, turned to be also effective in the case of zolpidem. This drug is structurally unrelated to benzodiazepine, but its pharmacological mechanism is similar: it binds to the omega- 1 subclass of benzodiazepine receptors (GABA-A) [7,8]. Flumazenil binds the GABA-A receptors at the same binding site for zolpidem and displaces it, decreasing its hypnotic effect [9].

From the analytic point of view, the restricted field of diagnostic applicability/accuracy of rapid tests performed in emergency (point of care and immunochemical tests), and the consequent necessity of a general unknown toxicological screening, performed according to sound toxicology guidelines, must be highlighted [10]. As a matter of fact, the psychotropic substances that are used to produce or selfinduce impairment can be different from the "classical" drugs of abuse covered by immunoassays in urine (cocaine, cannabinoids, opiates, methadone, amphetamines, benzodiazepines and barbiturates). In particular, new psychoactive drugs, either medicinal or illicit drugs, are on the market and demand surveillance. However, the clinical pathology laboratories are rarely equipped with gas-chromatography or liquid-chromatography coupled to specific detectors such as mass spectrometers $[11,12]$, and this calls for a collaboration with toxicology or forensic toxicology departments.

\section{Conclusions}

Physicians should consider DFCs in every case of altered mental status or anterograde amnesia lacking an evident clinical reason. In order to make a diagnosis, it is important to accurately collect medical history and perform a thorough physical examination, blood and radiographic exams and toxicological analysis. However, toxicological analysis performed in emergency are limited by a restricted field of diagnostic applicability/accuracy. The application of diagnostic procedures with more specificity/ sensibility, typical of the forensic toxicological setting, should prevent diagnostic delay and should avoid exams, such as cerebral $\mathrm{CT}$, that suffer of potential side effects and are more expensive than a toxicological analysis.

\section{Contributors}

FP wrote the case report, FDR and DF discussed the case, GC reviewed the literature, MT was involved in the toxicological analysis. 


\section{Patient Consent}

The case report is fully anonymised. The patient gave consent to blood drawing for clinical purposes.

\section{References}

1. Shbair MK Lhermitte M (2010) Drug-facilitated crimes: definitions, prevalence, difficulties and recommendations. A review. Ann Pharm Fr 68 : 136-147.

2. LeBeau MA, Montgomery MA (2010) Challenges of Drug-Facilitated Sexual Assault. Forensic Sci Rev 22: 1-6.

3. LeBeau, MA (2010) Laboratory management of drug-facilitated sexual assault cases. Forensic Sci Rev 22: 113-119.

4. Pépin G (2010) Analytical, toxicological and forensic aspects of drug facilitated crimes: 10 years of experience. Ann Pharm Fr 68: 61-75.

5. Forrester MB (2009) Immediate- and controlled-release zolpidem ingestions reported to Texas poison centers. Hum Exp Toxicol 28: 505-509.

6. Forrester MB (2006) Comparison of zolpidem and zaleplon exposures in Texas, 1998-2004. J Toxicol Environ Health A 69: 1883-1892.
7. Gunja N (2013) In the Zzz zone: the effects of Z-drugs on human performance and driving. J Med Toxicol 9: 163-171.

8. Crestani F, Martin JR, Möhler H, Rudolph U (2000) Mechanism of action of the hypnotic zolpidem in vivo. Br J Pharmacol 131: 1251-1254.

9. Johnston GA (1996) GABAA receptor pharmacology. Pharmacol Ther 69 173-198.

10. (2011) Guidelines for the Laboratory and Scientific Section, United Nations Office on Drugs and Crime, Vienna. Guidelines for the Forensic analysis of drugs facilitating sexual assault and other criminal acts. New York 2011. Laboratory and Scientific Section, United Nations Office on Drugs and Crime, Vienna, New York.

11. Versace F, Sporkert F, Mangin P, Staub C (2012) Rapid sample pre-treatment prior to GC-MS and GC-MS/MS urinary toxicological screening. Talanta 101: 299-306

12. Ojanperä I, Kolmonen M, Pelander A (2012) Current use of high-resolution mass spectrometry in drug screening relevant to clinical and forensic toxicology and doping control. Anal Bioanal Chem 403: 1203-1220. 